Check for updates

Cite this: RSC Adv., 2019, 9, 10766

Received 29th January 2019

Accepted 31st March 2019

DOI: 10.1039/c9ra00770a

rsc.li/rsc-advances

\section{Comparative effects of mercury chloride and methylmercury exposure on early neurodevelopment in zebrafish larvae}

\author{
Jun Zhu, $\dagger^{\mathrm{a}}$ Chundan Wang,,$^{\mathrm{a}}$ Xingsu Gao, ${ }^{\mathrm{a}}$ Jiansheng Zhu, ${ }^{\mathrm{b}} \mathrm{Li}$ Wang, ${ }^{\mathrm{a}}$ Shuyuan Cao, ${ }^{\mathrm{a}}$ \\ Qian Wu, ${ }^{a}$ Shanlei Qiao, ${ }^{\text {a }}$ Zhan Zhang (iD) *a and Lei Li (iD *a
}

Mercury $(\mathrm{Hg})$ is a ubiquitous environmental toxicant with important public health implications. $\mathrm{Hg}$ causes neurotoxicity through astrocytes, $\mathrm{Ca}^{2+}$, neurotransmitters, mitochondrial damage, elevations of reactive oxygen species and post-translational modifications. However, the similarities and differences between the neurotoxic mechanisms caused by different chemical forms of $\mathrm{Hg}$ remain unclear. Zebrafish embryos were exposed to methylmercury $(\mathrm{MeHgCl})$ or mercury chloride $\left(\mathrm{HgCl}_{2}\right)(0,4,40,400 \mathrm{nM})$ up for $96 \mathrm{~h}$. $\mathrm{HgCl}_{2}$ exposure could significantly decrease survival rate, body length and eye size, delay the hatching period, induce tail bending and reduce the locomotor activity, and these effects were aggravated in the $\mathrm{MeHgCl}$ group. The compounds could increase the number of apoptotic cells in the brain and downregulate the expression of Shha, Ngn1 and Nrd, which contribute to early nervous development. The underlying mechanisms were investigated by metabolomics data. Galactose metabolism, tyrosine metabolism and starch and sucrose metabolism pathways were disturbed after $\mathrm{HgCl}_{2}$ or $\mathrm{MeHgCl}$ exposure. In addition, the levels of three neurotransmitters including tyrosine, dopamine and tryptophan were reduced after $\mathrm{HgCl}_{2}$ or $\mathrm{MeHgCl}$ exposure. Oxidative stress is related to metabolite changes, such as changes in the putrescine, niacinamide and uric acid contents in the $\mathrm{HgCl}_{2}$ group, and squalene in the $\mathrm{MeHgCl}$ group. These data indicated that downregulation of these genes and abnormal metabolic profile and pathways contribute to the neurotoxicity of $\mathrm{HgCl}_{2}$ and $\mathrm{MeHgCl}$.

\section{Introduction}

Mercury ( $\mathrm{Hg}$ ) is severely detrimental to organisms and is ubiquitous in both terrestrial and aquatic ecosystems. ${ }^{1}$ It is released from a variety of anthropogenic, industrial processes, enters the Earth's atmosphere and is re-deposited onto the Earth's surface in rainfall. ${ }^{2}$ In natural systems, mercury is found in elemental, inorganic, and organometallic forms. The environmental emission of mercury could reach up to 8900 tons annually, of which $90 \%$ consist of anthropogenic emissions from processes such as artisanal gold mining. ${ }^{3}$ Unfortunately, China is the largest emitter of $\mathrm{Hg}$, responsible for $25 \%$ of the world's mercury emissions. ${ }^{4}$ Estimated annual sedimentary fluxes of mercury in the East China Sea are $5.1 \times 10^{7} \mathrm{~g}$, which accounts for $9 \%$ of China's annual mercury emissions. ${ }^{5}$ A recent

${ }^{a}$ Center for Global Health, School of Public Health, Nanjing Medical University, 101 Longmian Avenue, Nanjing, Jiangsu 211166, P. R. China. E-mail: drleili@hotmail. com; zhanzhang@njmu.edu.cn; alexqiao@139.com; Fax: +86-25-8686-8499; Tel: +86-25-8686-8402; +86-25-8686-8404

${ }^{b}$ Key Lab of Modern Toxicology of Ministry of Education, School of Public Health, Nanjing Medical University, 101 Longmian Avenue, Nanjing, Jiangsu 211166, P. R. China

$\dagger$ These authors contributed equally to this work. study has shown that the concentration of total $\mathrm{Hg}$ in untreated sewage was as high as $15 \pm 4.4 \mu \mathrm{g} \mathrm{L}^{-1}$ and $\mathrm{MeHg}$ concentration was $17 \pm 1.8 \mathrm{ng} \mathrm{L^{-1 }}$ in Tibet, China. ${ }^{6}$ Worse still, it can bioaccumulate through the food chain. The concentrations of total $\mathrm{Hg}$ and $\mathrm{MeHg}$ were 0.5-272 $\mathrm{ng} \mathrm{g}^{-1}$ and 0.1-199 $\mathrm{ng} \mathrm{g}^{-1}$ (wet weight) in fish muscle from the Three Gorges Reservoir, respectively. ${ }^{7}$ The concentration of $\mathrm{MeHg}$ in fresh shellfish was $0.46-6.38 \mu \mathrm{g} \mathrm{kg}^{-1}$ in the typical coastal cities (Lianyungang and Yantai) in China. ${ }^{8}$

Mercury is a well-known teratogenic and neurotoxin to wildlife and humans. However, the toxicity, absorption and distribution of mercury vary among different forms, such as the metallic element, inorganic salts and organic compounds. ${ }^{9}$ Among them, methylmercury (MeHg) has attracted much attention due to its strong toxicity and ability to pass through biofilms. ${ }^{10}$ Previous studies had shown on the mechanism of MeHg-induced neurotoxicity, such as astrocytes, $\mathrm{Ca}^{2+}$, neurotransmitters, mitochondrial damage, elevations of reactive oxygen species and post-translational modifications, etc. ${ }^{\mathbf{1 1}}$ And inorganic mercury caused cell death by cytotoxicity and induction of apoptosis with a decreased number of neurons and astrocytes in the motor cortex. ${ }^{12}$ However, the interpretation of their neurotoxic mechanisms from metabolites was undefined. 
Metabolomics is a rapidly evolving technology for identifying and quantifying all metabolites that are present in a biological sample and might provide mechanistic insights into the etiology of various psychiatric disorders. ${ }^{13}$ Metabolomics analysis indicated that paraquat exposure induced alteration in the metabolites, which may lead to oxidative stress and neurodegeneration along with movement disorder in Drosophil. ${ }^{14}$ The integration of metabolomics and oxidative stress endpoints allowed the identification of reduced glutathione as a first line of defense against $\mathrm{Hg}$ in fish. ${ }^{15}$ Nuclear magnetic resonance (NMR)-based metabolomics revealed that exposure of $\mathrm{Hg}$ metabolites reduced glutathione and its constituent amino acids, glutamate and glycine in fish. ${ }^{16} \mathrm{MeHg}$ could also change metabolome, including lipid metabolism and neurotransmission in mouse brain and larval fish. ${ }^{17}$ Therefore, metabolomics is an efficient approach for pre-screening of environmental chemicals, such as $\mathrm{Hg}$ and $\mathrm{MeHg}$, for their neurodegenerative potential with altered metabolism.

In recent years, larval stage zebrafish have been emerging as a standard vertebrate model in a number of fields, ranging from developmental biology to pharmacology and toxicology. It has been recognized as a privileged vertebrate model due to its small size, wide distribution, easy growth conditions offering the possibility to perform high-throughput analysis for metabolic profiling and relative genetic proximity to humans. ${ }^{18,19}$ The differences between the neurotoxic mechanisms caused by different chemical forms of $\mathrm{Hg}$ in zebrafish remain unclear. The present study integrated the metabolomics and conventional neurodevelopmental endpoints to reveal the neurotoxicity and related mechanism of $\mathrm{HgCl}_{2}$ and $\mathrm{MeHg}$ in zebrafish larvae.

\section{Materials and methods}

\subsection{Reagents}

Methylmercury chloride ( $\mathrm{MeHgCl}, 95 \%$ purity) and $\mathrm{N}, \mathrm{O}$-bis(trimethylsilyl) trifluoroacetamide (BSTFA) were purchased from Sigma Aldrich (St. Louis, MO, USA). Mercury chloride $\left(\mathrm{HgCl}_{2}\right.$, 95\% purity) was obtained from Xiya Chemical Reagent Co., Ltd. (Shandong, China). HPLC grade acetonitrile and isopropanol were all purchased from Merck (Darmstadt, Germany). Deionized water was manufactured by Milli-Q50SP Reagent system (Millipore Corporation, MA, USA). The acridine orange (AO) kits and MS-222 reagent was obtained from YSY Biotech Co., Ltd. (Nanjing, China). Trizol reagent was obtained from Invitrogen (Carlsbad, CA, USA).

\subsection{Zebrafish maintenance and embryo collection}

Adult wild-type zebrafish (AB strain) were obtained from Nanjing YSY Biotech Company Ltd. Adult fish were housed at $28^{\circ} \mathrm{C}$ on a $14: 10$ light-dark photoperiod under semi-static conditions with charcoal filtered water. Zebrafish embryos were procured from zebrafish adults of wild type strain. Embryos were collected from spawning adult fish after 0.5 hour post fertilization (hpf). Embryos from different adult pairs were pooled in order to avoid batch effects. After collection, embryos were rinsed in egg water and maintained at $28 \pm 0.5^{\circ} \mathrm{C}$ in an incubator until $6 \mathrm{hpf}$, at which time exposure was initiated.

\subsection{Mercury preparation and exposure}

This study was performed in strict accordance with the NIH guidelines for the care and use of laboratory animals (NIH publication no. 85-23 rev. 1985) and was approved by the Animal Care and Welfare Committee of Nanjing Medical University (Nanjing, China). $\mathrm{MeHgCl}$ and $\mathrm{HgCl}_{2}$ were initially dissolved in sterile deionized water to a concentration of 400 $\mu \mathrm{M}$ respectively and further diluted with embryo medium for zebrafish embryos exposure. All stock solutions were stored at $4{ }^{\circ} \mathrm{C}$ until used. Zebrafish embryos were exposed to different concentrations of $\mathrm{MeHgCl}$ or $\mathrm{HgCl}_{2}$ in 6-well plates. Briefly, normal embryos at $6 \mathrm{hpf}$ were distributed randomly into well containing $30 \mathrm{~mL}$ of $\mathrm{MeHgCl}(0,4,40,400 \mathrm{nM})$ or $\mathrm{HgCl}_{2}(0,4$, $40,400 \mathrm{nM}$ ) solutions. Six independent experiments were performed with 60 embryos per experiment (total 360 embryos per group). After $24 \mathrm{~h}$ exposure to each concentration, all surviving embryos were transferred to fresh embryo medium without mercury. Survival and hatching rates of embryos and larvae were observed at intervals of $12 \mathrm{hpf}$ up to $96 \mathrm{hpf}$. At $30 \mathrm{hpf}, 20$ larvae each group were collected for GC-TOF-MS analysis and 30 larvae each group were collected for polymerase chain reaction (PCR) detection. While locomotor activity measurement and analysis of apoptosis were performed for each group of 12 larvae at $96 \mathrm{hpf}$.

\subsection{Morphological analysis of developmental neurotoxicity}

12 embryos per group were photographed from the side, and the central nervous system (CNS) anomalies were evaluated at $96 \mathrm{hpf}$ according to the previously reported methods. ${ }^{20}$ Briefly, morphological analysis of neurotoxicity was performed by measuring body length and eye size. The body length was measured horizontally from the foremost mouth to the tip of the tail. The eye size was examined when both eyes were completely overlapped. All measurements were performed using Nikon optical microscope (Nikon SMZ18, Japan).

\subsection{Locomotor activity measurement}

Locomotor activity was monitored and analyzed by Noldus system with Eth0VIsion XT software (DanioVisio, Noldus (Beijing) Information Technology Co., Ltd, Netherlands). Larval swimming behavior was monitored in response to dark-to-light transitions. The software program: firstly, giving the larval $5 \mathrm{~min}$ for adaptation period, then opening the automatic light to stimulate $10 \mathrm{~min}$, dark $10 \mathrm{~min}$, and the light and dark alternate process cycle 3 times. The data (frequency of movements, distance traveled, and total duration of movements) were collected every $20 \mathrm{~s}$. Locomotor behavior was monitored in a total of 12 larvae each group.

\subsection{Analysis of apoptosis in brain using acridine orange (AO)}

Zebrafish exposed for $95 \mathrm{hpf}$ were transferred to an $\mathrm{AO}$ solution diluted to $5 \mu \mathrm{g} \mathrm{mL} \mathrm{m}^{-1}$ in embryo medium and incubated for $1 \mathrm{~h}$ 
without light. Subsequently, AO was removed and the zebrafish washed several times with fresh embryo medium were examined. Before examination, the larvae were anesthetized with $0.03 \%$ MS-222 for $1 \mathrm{~min}$. Stained larvae were photographed under a fluorescent stereo microscope (Nikon SMZ18, Japan). The apoptotic cells appear overt bright spots. ${ }^{21} 12$ embryos per group were analyzed and the number of cells showing acridine orange positivity in the brain region was compared between groups.

\subsection{Total RNA isolation and quantitative real-time PCR analysis}

Total RNA was extracted from 30 living zebrafish larvae (30 hpf) treated with $\mathrm{HgCl}_{2}$ or $\mathrm{MeHgCl}$ using Trizol reagent. The expression of early neurogenesis related genes were measured by realtime polymerase chain reaction (PCR). The primers were synthesized by GenScript Biotech Corp. (Nanjing, China): Sonic hedgehog a (Shha) (forward AGACCGAGACTCCACGACGC and reverse, TGCAGTCACTGGTGCGAACG), Neurogenin1 (Ngn1) (forward TGCACAACCTTAACGACGCATTGG and reverse, TGCCCAGATGTAGTTGTGAGCGAA) and Neuro D (Nrd) (forward CAGCAAGTGCTTCCTTTTCC and reverse, TAAGGGGTCCGTCAAATGAG) and $\beta$-actin (forward ATGGATGAGGAAATCGCTGCC and reverse, CTCCCTGATGTCTGGGTCGTC). PCR was performed in $10 \mu \mathrm{L}$ reaction volume on a LightCycler® 96 Detection System (Roche Diagnostics (Shanghai) Ltd., Switzerland).

\subsection{Sample preparation and GC-TOF-MS analysis}

At $30 \mathrm{hpf}, 20$ larvae per replicate were strained and washed several times with egg water to remove any bound particulates or chemical residues attached to body surfaces. Next, the 20 larvae were transferred into $1.5 \mathrm{~mL}$ centrifuge tubes and chilled on ice to anesthetize and suppress enzymatic activity. $10 \mu \mathrm{L}$ of the two internal standard, $p$-chlorophenylalanine $\left(0.1 \mathrm{mg} \mathrm{L}^{-1}\right)$ and heptadecanoic acid $\left(1 \mathrm{mg} \mathrm{mL}^{-1}\right)$, as well as $1 \mathrm{~mL}$ of the cold extraction solvent (acetonitrile : isopropanol : water, $3: 3: 2 ; \mathrm{v} /$ $\mathrm{v} / \mathrm{v}$ ) mixture was added to each sample on ice. The tubes were vortexed for $30 \mathrm{~s}$. Afterwards, the sample was homogenized in a ball mill for $30 \mathrm{~min}$ at $30 \mathrm{hz}$ while being incubated in ice water. $150 \mu \mathrm{L}$ liquid from each tube was pooled as quality control (QC) solution after centrifugation at $13000 \mathrm{rpm}$ for $15 \mathrm{~min}$ at $4{ }^{\circ} \mathrm{C}$. Then, an aliquot of $800 \mu \mathrm{L}$ of supernatant was transferred into a fresh 2 mL GC-TOF-MS glass vial and dried in a vacuum concentrator. The residue was derivatized by addition of $80 \mu \mathrm{L}$ of methoxyamine hydrochloride $\left(15 \mathrm{mg} \mathrm{mL}^{-1}\right.$ in pyridine) incubated for $90 \mathrm{~min}$ at $37^{\circ} \mathrm{C}$, followed by the addition of $50 \mu \mathrm{L}$ BSTFA and incubated for $60 \mathrm{~min}$ at $70{ }^{\circ} \mathrm{C}$.

According to previous study, ${ }^{22}$ trimethylsilyl derivatives can be stable for $30 \mathrm{~h}$ after derivatization. $1 \mu \mathrm{L}$ of solution was immediately injected into an Agilent 7890 gas chromatograph system (Agilent Corporation, Santa Clara, CA, USA) coupled with a Leco Pegasus time-of-flight mass spectrometer (GC-TOF-MS) in splitless mode. The system was equipped with a DB-5MS capillary column $(30 \mathrm{~m} \times 0.25 \mathrm{~mm}$ inner diameter, $0.25 \mu \mathrm{m}$ film thickness; J\&W Scientific, Folsom, CA, USA). Helium was used as the carrier gas, with a constant flow rate of $1 \mathrm{~mL} \mathrm{~min}{ }^{-1}$.
The temperature program was set up as follows: the initial temperature was kept at $50{ }^{\circ} \mathrm{C}$ for $2 \mathrm{~min}$, then elevated to $180{ }^{\circ} \mathrm{C}$ at a rate of $10{ }^{\circ} \mathrm{C} \mathrm{min}^{-1}$, then elevated to $240{ }^{\circ} \mathrm{C}$ at a rate of 5 ${ }^{\circ} \mathrm{Cmin}^{-1}$, then elevated to $290{ }^{\circ} \mathrm{C}$ at a rate of $25{ }^{\circ} \mathrm{Cmin}^{-1}$ and sustained for $10 \mathrm{~min}$ at $290^{\circ} \mathrm{C}$. The temperatures of the injector, transfer line and ion source were set to 270,260 and $240{ }^{\circ} \mathrm{C}$, respectively. A mass range of $50-550 \mathrm{~m} / \mathrm{z}$ in full-scan mode for electron impact ionization $(70 \mathrm{eV})$ was applied. The solvent delay time was set to $7.33 \mathrm{~min}$.

\subsection{Statistical analyses}

The differences in toxic effects, morphological alterations related to developmental neurotoxicity, gene expression and metabolite change were analyzed using one-way analysis of variance (ANOVA) by SPSS 13.0 software (Chicago, IL, USA). The Chroma TOF4.3X software of LECO Corporation and LECOFiehn Rtx5 database were used for raw peak extracting, data baselines filtering and calibration of the baseline, peak alignment, deconvolution analysis, peak identification and integration of the peak area. The statistical significance was calculated using the Student $t$-test. Metabolic data were analyzed using $\mathrm{R}$ to screen for metabolic differences. Differences were considered statistically significant at $P \leq 0.05$, after a fdr correction by " $\mathrm{BH}$ " method. R package "pheatmap" is used to draw heat maps. The free web-based tool MetaboAnalyst 4.0 uses a high-quality KEGG metabolic pathway (Danio rerio) database as a back-end knowledge base for pathway analysis and visualization (http:// www.metaboanalyst.ca).

\section{Results}

\subsection{Effects of $\mathrm{HgCl}_{2}$ and $\mathrm{MeHgCl}$ on the embryonic development in zebrafish}

The survival rate significantly decreased at $48 \mathrm{hpf}$ when the zebrafishes were exposed to $400 \mathrm{nM} \mathrm{HgCl}_{2}$ or $40 \mathrm{nM} \mathrm{MeHgCl}$. At $96 \mathrm{hpf}$, survival rate in $400 \mathrm{nM} \mathrm{HgCl}$ and $400 \mathrm{nM} \mathrm{MeHgCl}$ were $77.54 \pm 4.53 \%(P=0.0009)$ and $75.36 \pm 5.02 \%(P=0.0003)$ of control, respectively. There were about $5 \%$ dead in the control

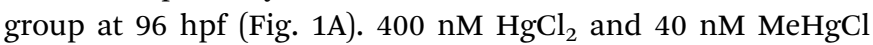
could significantly delay the zebrafish hatching at $60 \mathrm{hpf}$ and 72 hpf (Fig. 1B). The tail bending was observed in $40 \mathrm{nM} \mathrm{HgCl}_{2}$ and $40 \mathrm{nM} \mathrm{MeHgCl}$ at $96 \mathrm{hpf}$ (Fig. 1C). The body length in $400 \mathrm{nM}$ $\mathrm{HgCl}_{2}$ and $400 \mathrm{nM} \mathrm{MeHgCl}$ group were $87.58 \pm 7.2 \%(P=$ $0.0001)$ and $92.16 \pm 9.50 \%(P=0.0001)$ of control, respectively (Fig. 1D). The eye size decreased significantly to $64.79 \pm 12.02 \%$ $(P=0.0001)$ and $75.96 \pm 15.86 \%(P=0.0001)$ in $400 \mathrm{nM} \mathrm{HgCl}_{2}$ and $400 \mathrm{nM} \mathrm{MeHgCl}$, respectively (Fig. 1E).

\subsection{Effects of $\mathrm{HgCl}_{2}$ and $\mathrm{MeHgCl}$ on the locomotor activity of zebrafish}

$\mathrm{MeHgCl}$ exposure could significantly decrease the average moving distance, while this effect was not observed after $\mathrm{HgCl}_{2}$ exposure (Fig. 2A and $\mathrm{C}$ ). The trajectory disturbance was observed in $\mathrm{HgCl}_{2}$ or $\mathrm{MeHgCl}$ group at the concentration of $40 \mathrm{nM}$ or $400 \mathrm{nM}$, reflecting increased traces in the middle area (Fig. 2B). 
A

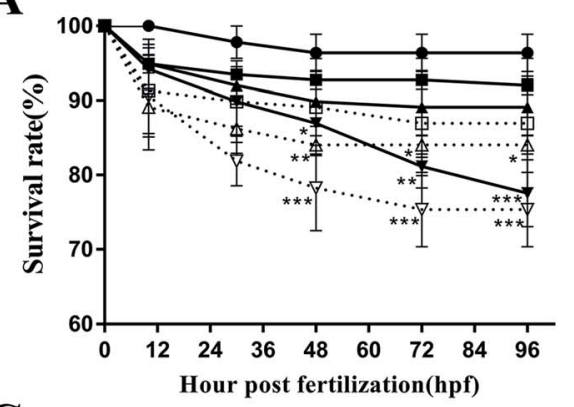

C

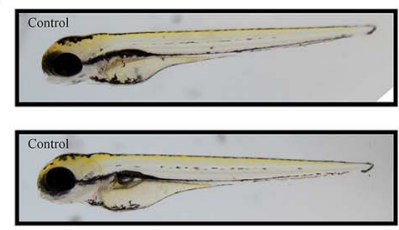

D

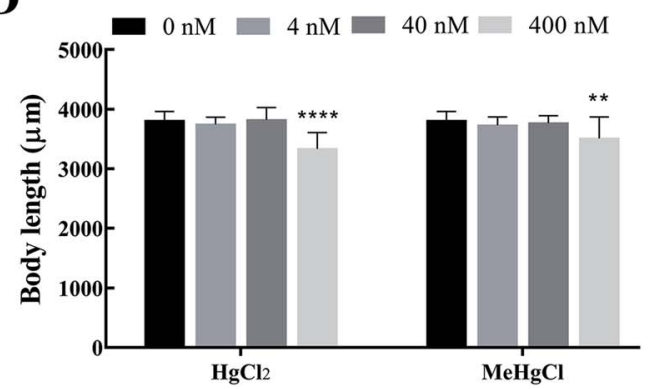

B
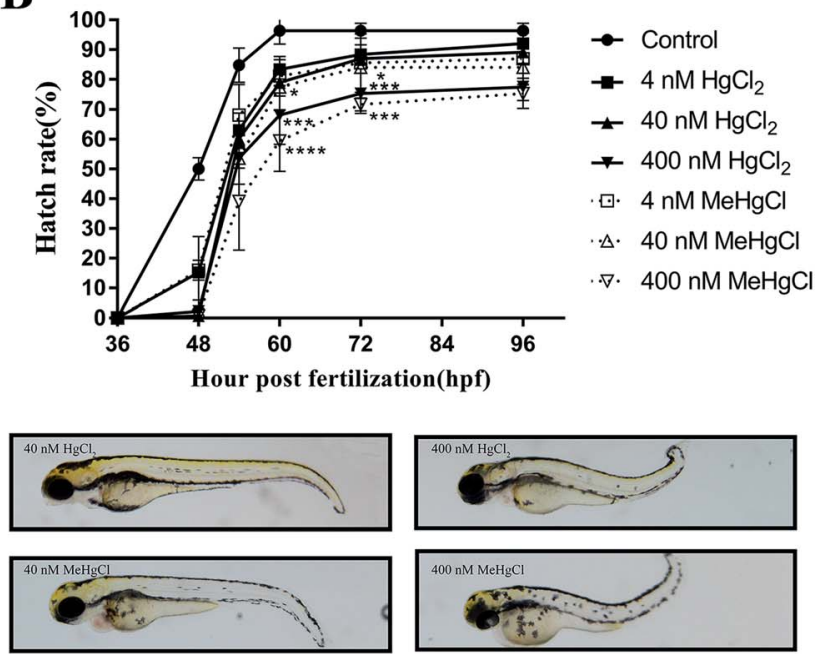

E

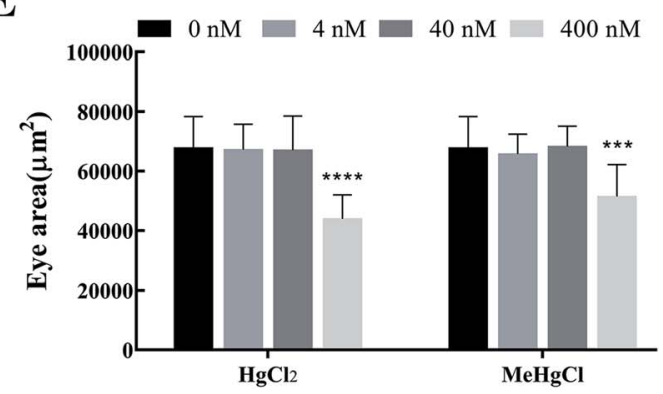

Fig. 1 Effects of mercury chloride $\left(\mathrm{HgCl}_{2}\right)$ and methylmercury chloride $(\mathrm{MeHgCl})$ on the embryonic development. Zebrafish embryos were exposed to $\mathrm{MeHgCl}$ (control, 4, 40, $400 \mathrm{nM}$ ) and $\mathrm{HgCl}_{2}$ (control, 4, 40, $400 \mathrm{nM}$ ) for up to 96 hpf. (A) Survival rates, (B) hatching rates, (C) morphological abnormalities, (D) body length, (E) eye size of embryos were investigated. Data represent the mean $\pm \mathrm{SD}$ of three experiments. $* P$ $<0.05 ; * * P<0.01, * * * P<0.001, * * * * P<0.0001$; compared with control group.

\subsection{Effects of $\mathrm{HgCl}_{2}$ and $\mathrm{MeHgCl}$ on brain cell apoptosis and the expression of neural development related genes}

Acridine orange staining was performed to detect the apoptosis in the brain. $8.25 \pm 1.86$ positive cells were detected in control. There were $20.08 \pm 3.68$ and $27.5 \pm 6.43$ positive cells in the brain of zebrafish exposed to $40 \mathrm{nM}$ and $400 \mathrm{nM} \mathrm{HgCl}_{2}$, respectively. Compared with $\mathrm{HgCl}_{2}, 400 \mathrm{nM} \mathrm{MeHgCl}$ induced more apoptotic cell $(34.42 \pm 6.16)$ (Fig. $3 \mathrm{~A}$ and B). The expression of $S h h, N g n 1$ and $N r d$ were significantly downregulated by $\mathrm{HgCl}_{2}$ and $\mathrm{MeHgCl}$ even at $4 \mathrm{nM}$ (Fig. 3C-E).

\subsection{Metabolite profile changes}

There 2369 metabolite peaks were routinely identified in extracted zebrafish embryo samples. After deleting RSD $>30 \%$ in QC samples and the unnamed peak, 15 metabolites were identified $(P$ $<0.05$ ) significantly different between $40 \mathrm{nM} \mathrm{HgCl}_{2}$ and control group, and 13 significantly different metabolite peaks in $40 \mathrm{nM}$ $\mathrm{MeHgCl}$ group (Table 1). The heatmap was developed based on normalized data using autoscale features for standardization (Fig. 4A and B). Pathway analysis revealed perturbation of five main pathways by $\mathrm{HgCl}_{2}$, including (i) galactose metabolism, (ii) tyrosine metabolism, (iii) starch and sucrose metabolism (iv) phenylalanine, tyrosine and tryptophan biosynthesis and (v) alpha-linolenic acid metabolism. And for $\mathrm{MeHgCl}$, (i) tyrosine metabolism, (ii) starch and sucrose metabolism, (iii) galactose metabolism (iv) sphingolipid metabolism and (v) arachidonic acid metabolism (Fig. 4C and D) were disturbed. The 8 metabolites ( $\mathrm{L}^{-}$ tyrosine, dopamine, L-tryptophan, sphingosine, arachidonic acid, melibiose, sucrose, gentisic acid) were found in both groups (Table 1). Among them, tyrosine (Fig. 4E), dopamine (Fig. 4F) and tryptophan (Fig. 4G) were associated with the neurotransmitter system. In addition, uric acid, putrescine, docosahexaenoic acid, D-ribulose 5-phosphate, alpha-linolenic acid, niacinamide, and Dglucose were significantly changed upon $\mathrm{HgCl}_{2}$ exposure; while $\mathrm{L}^{-}$ dopa, urea, squalene, eicosapentaenoic acid and D-maltose were significantly changed in $\mathrm{MeHgCl}$ group.

\section{Discussion}

Mercury $(\mathrm{Hg})$ is ubiquitary, naturally enriched in volcanic regions, and has wide applications in science, industry, and agriculture. $\mathrm{MeHgCl}$ and $\mathrm{HgCl}_{2}$ are potent neurotoxins which present considerable risk to the health of marine food chains. $\mathrm{MeHg}$ is the main mercury species in fish, normally accounting for $90 \% .^{7}$ Relatively low total $\mathrm{Hg}$ and $\mathrm{MeHg}$ concentrations were found in the majority of the white rice samples from 15 provinces across China with an overall mean of $4.74 \mu \mathrm{g} \mathrm{kg}^{-1}$ and $0.682 \mu \mathrm{g} \mathrm{kg}{ }^{-1}$, respectively. ${ }^{23}$ The $\mathrm{MeHg}$ levels of the fish samples from Hongkong ranged from $<2-$ 
A

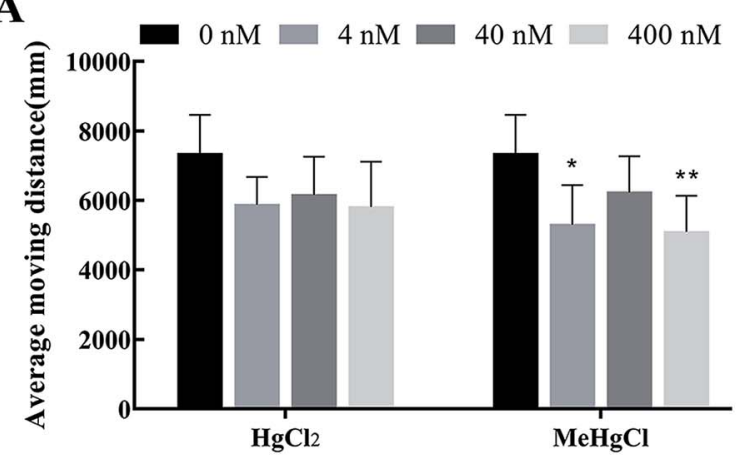

B

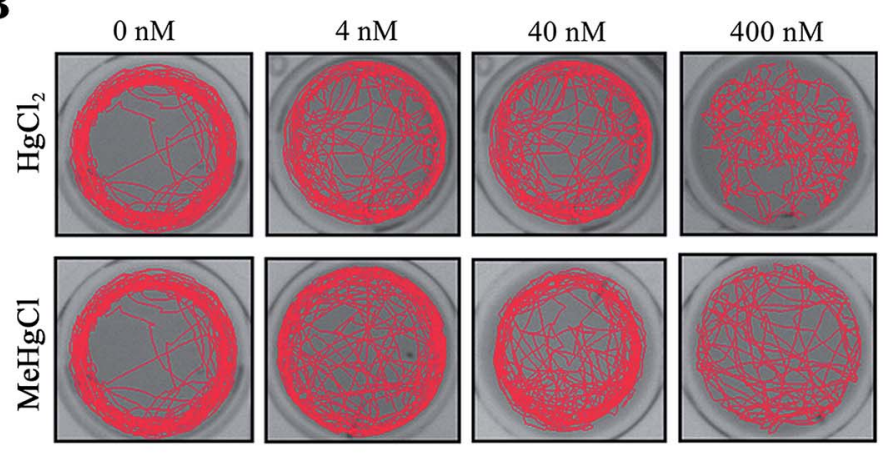

C

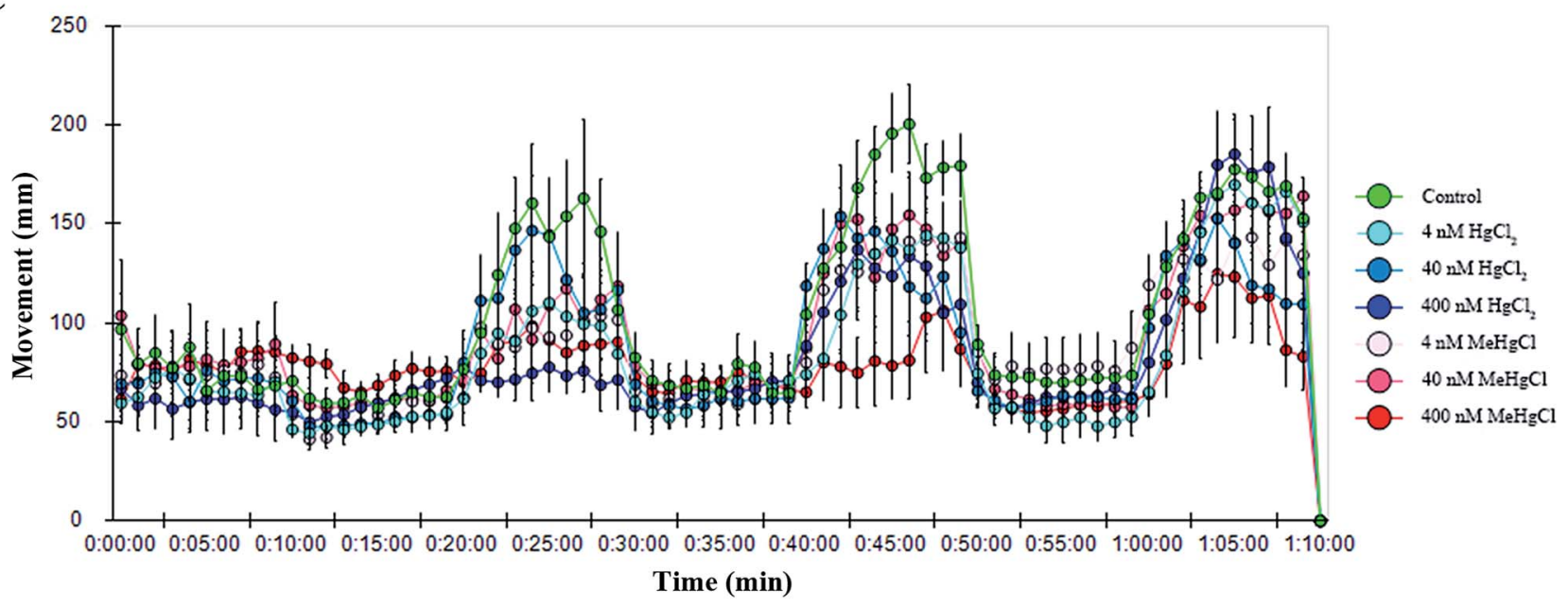

Fig. 2 Effects of mercury chloride $\left(\mathrm{HgCl}_{2}\right)$ and methylmercury chloride $(\mathrm{MeHgCl})$ on the locomotor activity. The embryos/larvae were exposed to $\mathrm{MeHgCl}$ (control, 4, 40, $400 \mathrm{nM}$ ) or $\mathrm{HgCl}_{2}$ (control, 4, 40, $400 \mathrm{nM}$ ), and their locomotor behavior were analyzed by the dark-light-dark photoperiod stimulation test at $96 \mathrm{hpf}$. (A) Average moving distance, (B) locomotor traces chart, (C) locomotor traces. * $P<0.05$; $* * P<0.01$; compared with control group.

$1498.7 \mathrm{ng} \mathrm{g}^{-1}$, and the median estimated MeHg intake for children and mothers were 0.29 and $0.22 \mu \mathrm{g}$ per $\mathrm{kg}$ bw per week, respectively. ${ }^{24}$ The concentration of total $\mathrm{Hg}$ in blood of bottlenose dolphins was $63.9 \pm 34.0 \mu \mathrm{g} \mathrm{L}^{-1}(319.5-489.5 \mathrm{nM}){ }^{2}$ which is near to $400 \mathrm{nM}$, the maximum dose in this study.

Numerous of evidence indicates that embryonic exposure to mercury can produce detrimental effects on neurocognitive functions in the children from infancy to adolescence. ${ }^{25}$ Neurotoxicity caused by different forms of mercury included the alteration of lipid peroxidation (LPO), $\mathrm{Na}^{+} / \mathrm{K}^{+}$-ATPase activities, and nitric oxide $\left(\mathrm{NO}_{x}\right)$ in the brain tissues from developing offspring mice. ${ }^{26}$ However, few studies compare the differences between the two. In the present study, zebrafish larvae was exposed to $\mathrm{HgCl}_{2}$ or $\mathrm{MeHgCl}$ for up to $96 \mathrm{~h}$. Both $\mathrm{HgCl}_{2}$ and $\mathrm{MeHgCl}$ exposure could decrease the survival rate, hatching rate and body length. $\mathrm{MeHgCl}$, but not $\mathrm{Hg}$ could significantly decreased the locomotor activity, reflecting more developmental toxicity than $\mathrm{Hg}$ in zebrafish. In addition, both $\mathrm{HgCl}_{2}$ and $\mathrm{MeHgCl}$ increased the apoptotic cells in zebrafish brain and downregulated the expression of Shha, Ngn1 and $N r d$, which regulate proliferation, differentiation and migration of neural progenitor and precursor cells. Then, metabolomics data was used to investigate metabolic signatures and underlying mechanism of neurotoxicity of mercury exposure.
In this study, both $\mathrm{MeHgCl}$ and $\mathrm{HgCl}_{2}$ could impair the embryonic development and locomotor activity, suggesting their neurotoxicity. This is supported by findings from another study where embryonic $\mathrm{MeHg}$ exposure induced learning impairments, persisting for at least three generations. ${ }^{27}$ Assessment of the developmental neurotoxicity of compounds by measuring locomotor activity in zebrafish embryos and larvae has become an alternative to developmental neurotoxicity testing. ${ }^{28}$ Although the nanomolar concentration of $\mathrm{HgCl}_{2}$ and $\mathrm{MeHgCl}$ used in this study, they show embryonic developmental toxicity. These development and locomotor activity data show that $\mathrm{HgCl}_{2}$ and $\mathrm{MeHgCl}$ may increase the permeability barrier and penetrate the chorion, which protect the developing embryo from the environmental toxins. ${ }^{28}$ In this study, $\mathrm{MeHgCl}$ exposure induced more apoptotic cells in the brain than those of $\mathrm{HgCl}_{2}$ at the same concentration, indicating that $\mathrm{MeHgCl}$ was more neurotoxic than $\mathrm{HgCl}_{2}$. Absorbed $\mathrm{MeHg}$ is assimilated through the gastrointestinal barrier, then passes into the blood where it is distributed into target organs, mainly brain and muscle. Chronic consumption of MeHg contaminated fish can cause adverse health effects in humans, especially on the central nervous and immune systems. ${ }^{29}$ In contrast, inorganic mercury tends to be accumulated in detoxification organs (liver and kidney)..$^{30}$ 
A
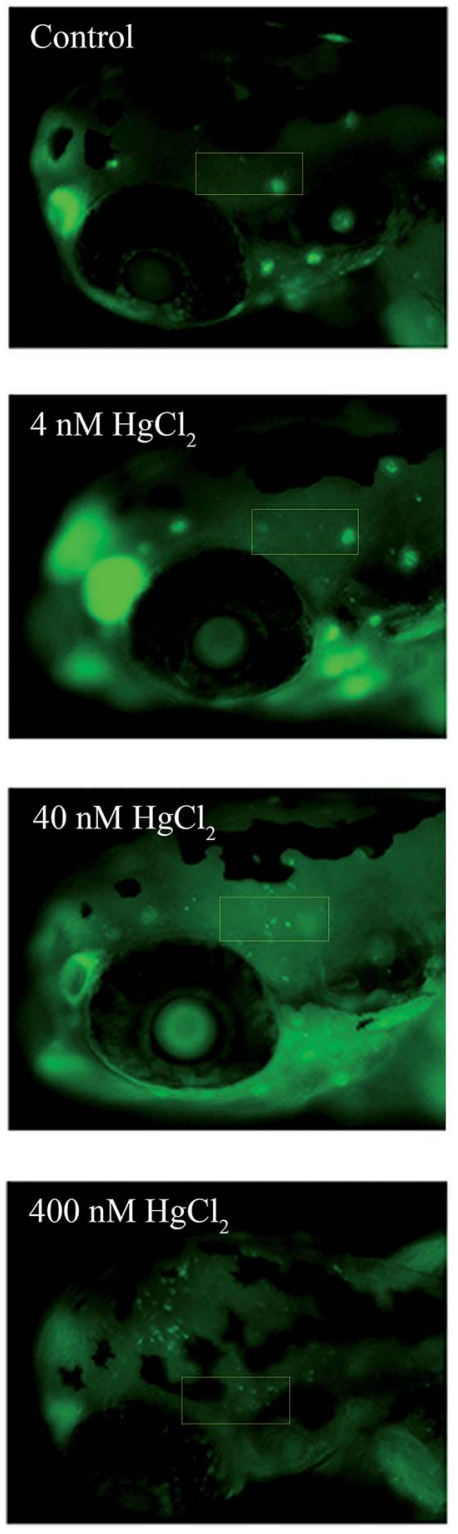
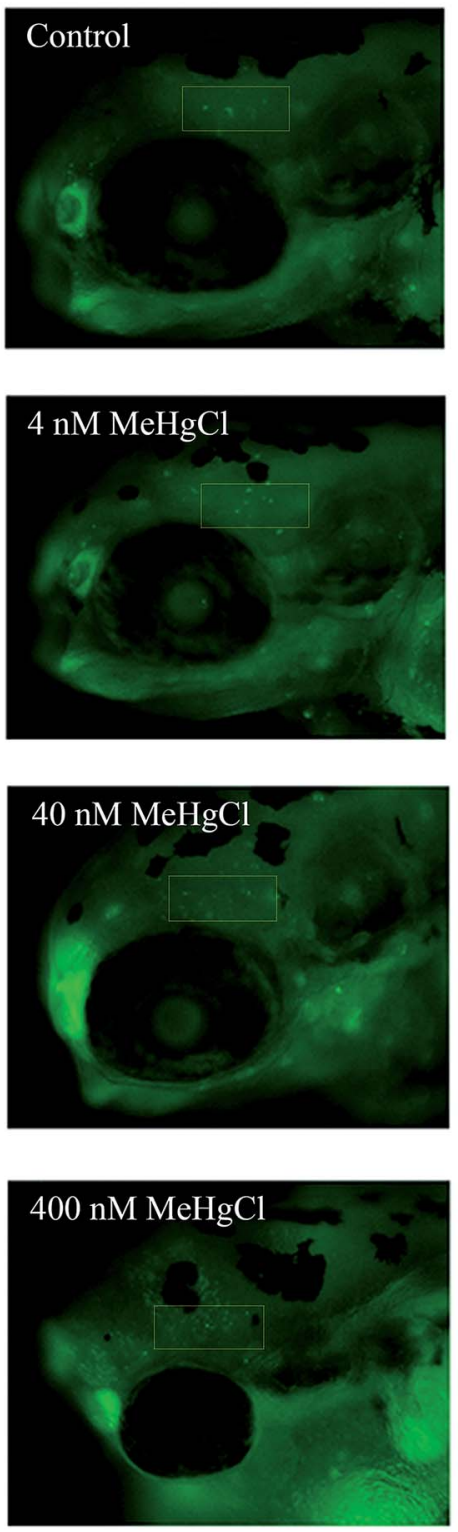

B

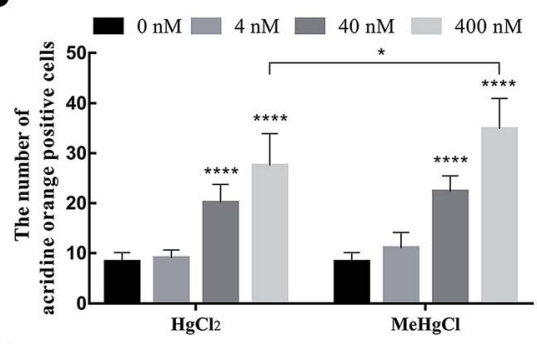

C

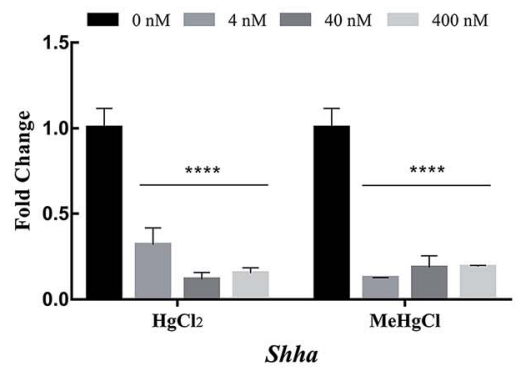

D

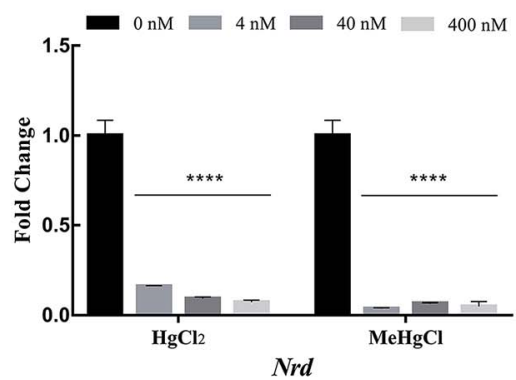

E

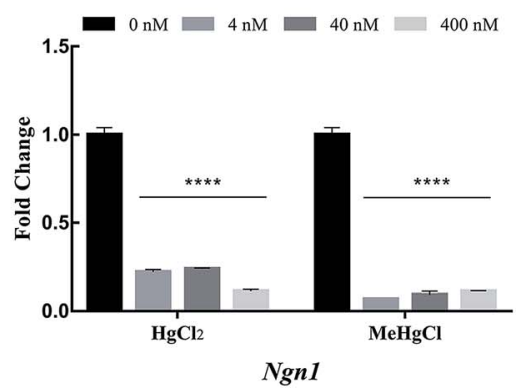

Fig. 3 Effects of mercury chloride $\left(\mathrm{HgCl}_{2}\right)$ and methylmercury chloride $(\mathrm{MeHgCl})$ on brain cell apoptosis and the expression of neural development related genes. The embryos/larvae were exposed to $\mathrm{MeHgCl}$ (control, 4, 40, $400 \mathrm{nM}$ ) or $\mathrm{HgCl}_{2}$ (control, 4, $40,400 \mathrm{nM}$ ), and the apoptotic cells in the brain were analyzed by acridine orange (AO) staining at $96 \mathrm{hpf}$. (A) Representative images of $\mathrm{AO}$ staining and (B) the number of $\mathrm{AO}$ positive cells in brain. $n=12$ embryos per group. The expression of (C) Shha, (D) Ngn1 and (E) Nrd in the zebrafish treated with $\mathrm{HgCl} 2$ and $\mathrm{MeHgCl}$ for $30 \mathrm{hpf}$. Data represent the mean $\pm \mathrm{SD}$. ${ }^{*} * P<0.01, * * * P<0.001, * * * * P<0.0001$; compared with control group.

Malformation is a common symptom in zebrafish exposed to environmental chemical exposure. Exposure of pyraoxystrobin triclosan or lead could result in malformed larvae exhibiting spinal curvature and bent tail. ${ }^{31,32}$ In the present study, exposure of $\mathrm{HgCl}_{2}$ or $\mathrm{MeHgCl}$ caused shortened body length, smaller eyes size and tail bending in zebrafish larvae. They also increased apoptotic cell in the brain, which may result in abnormal neurological development. The tail deformity is associated with early neurodevelopmental abnormalities. ${ }^{33}$ Furthermore, previous studies noted that increased apoptosis at this developmental stage is related to curled tail. ${ }^{34,35}$ In addition, abnormal neurological development may also impair the locomotor activity. ${ }^{36,37}$ Decreased movement distance after $\mathrm{HgCl}_{2}$ or
$\mathrm{MeHgCl}$ exposure may result from abnormal neurological development induced tail bending.

To investigate the mechanism of neurotoxicity of $\mathrm{HgCl}_{2}$ and $\mathrm{MeHgCl}$, the expression of three genes (Shha, Ngn1, Nrd) involving in early neural developmental stages was assessed. Shha controls key developmental processes, including neural tube patterns, neural stem cell proliferation, and survival of neurons and glial cells. ${ }^{38,39}$ Ngn is a marker for neuronal precursors and expressed in the central nervous system regulated by Shha. ${ }^{40} \mathrm{Ngn} 1$ is expressed in the neural plate and can induce the expression of Nrd, which may in turn cause proliferative neural precursor cells to become post-mitotic neurons. ${ }^{33}$ Trimethyltin chloride could inhibit neuronal cell 
Table 1 List of differential metabolites after $\mathrm{HgCl}_{2}$ or $\mathrm{MeHgCl}$ exposure at the concentration of $40 \mathrm{nM}^{a}$

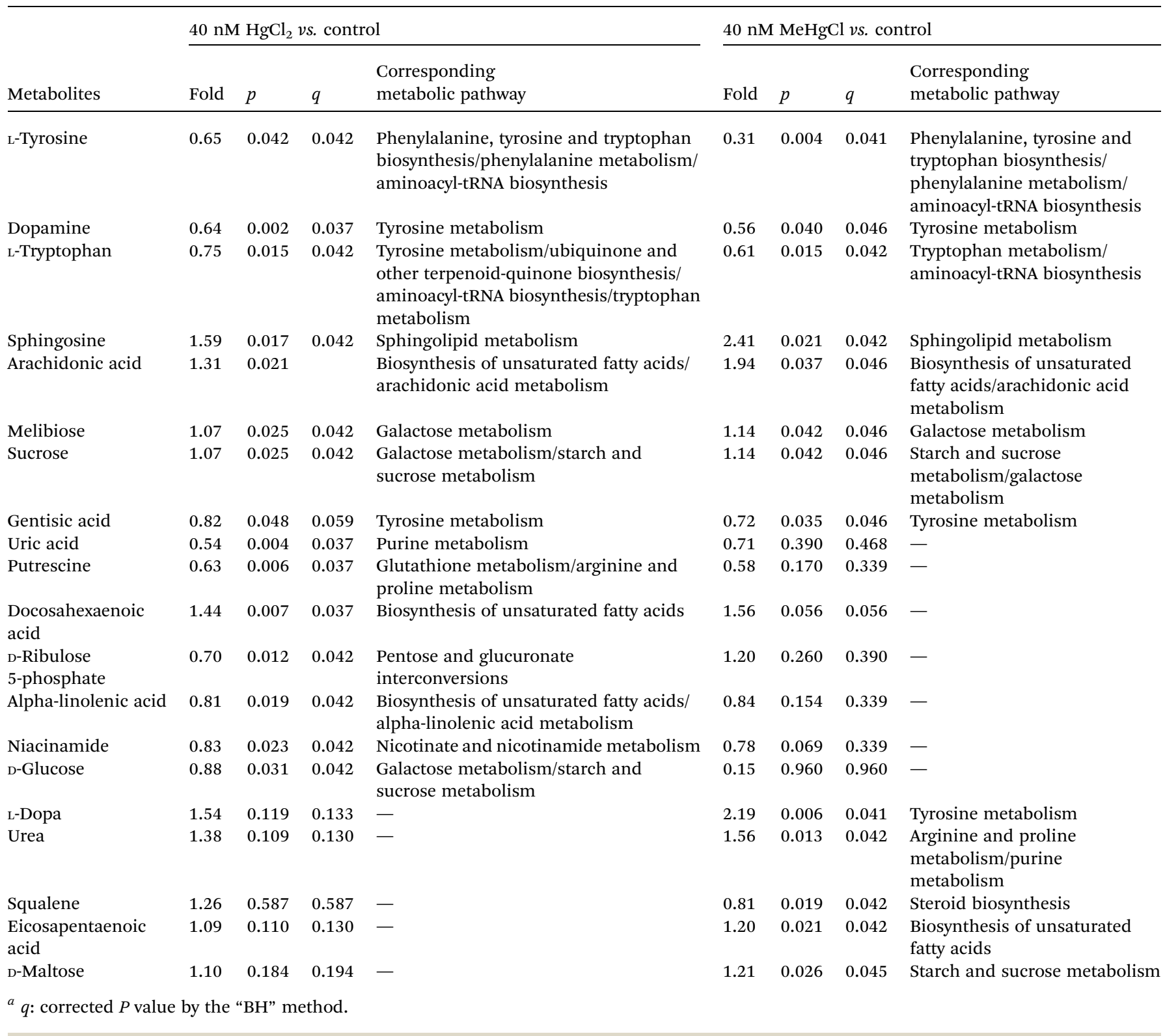

differentiation in zebrafish embryo neurodevelopment via upregulation of the expression of Shha and $N g n 1 .^{41}$ Aqueous cigarette tar extracts reduced the expression $\mathrm{Nrd}$ and affected cortical neuron development and neurodegeneration in human embryonic stem cells in vitro. ${ }^{42}$ In this study, $\mathrm{HgCl}_{2}$ and $\mathrm{MeHgCl}$ could significantly reduce the expression of Shha, $N g n 1$ and $N r d$ even at the concentration of $4 \mathrm{nM}$, suggesting that they could cause early developmental abnormalities of neurons and neural progenitors after exposure to the gastrula stage.

Characteristic metabolic deregulations of $\mathrm{HgCl}_{2}$ or $\mathrm{MeHgCl}$ exposure may enable novel mechanism discovery for their neurotoxicity. Galactose metabolism, tyrosine metabolism and starch and sucrose metabolism pathways were disturbed after $\mathrm{HgCl}_{2}$ or $\mathrm{MeHgCl}$ exposure. Phenylalanine, tyrosine and tryptophan biosynthesis pathway was also affected by $\mathrm{HgCl}_{2}$ exposure. Phenylalanine is a precursor of the amino acid tyrosine, which in turn causes the formation of neurotransmitters such as dopamine. ${ }^{43}$ Dopamine and serotonin are neuromodulators which contribute to associative learning performance in zebrafish. ${ }^{\mathbf{4 4}}$ In the present study, dopamine and tyrosine were decreased in the $\mathrm{HgCl}_{2}$ or $\mathrm{MeHgCl}$ group, indicating that neurotransmitter production may be affected. $\mathrm{HgCl}_{2}$ or $\mathrm{MeHgCl}$ exposure also decreased tryptophan, which is a neurotransmitter system belonging to serotonin. ${ }^{45}$ Intraspinal serotonergic signaling suppresses locomotor activity in larval zebrafish and chemogenetic ablation of dopaminergic neurons leads to transient locomotor impairments in zebrafish larvae. ${ }^{46,47}$ Putrescine, niacinamide, uric acid, and squalene all can induce oxidative stress, which may cause apoptosis and nerve damage. ${ }^{\mathbf{1 3} 48}$ But in $\mathrm{HgCl}_{2}$ group, putrescine, niacinamide, uric acid was observed changed, whereas squalene in $\mathrm{MeHgCl}$. 
A

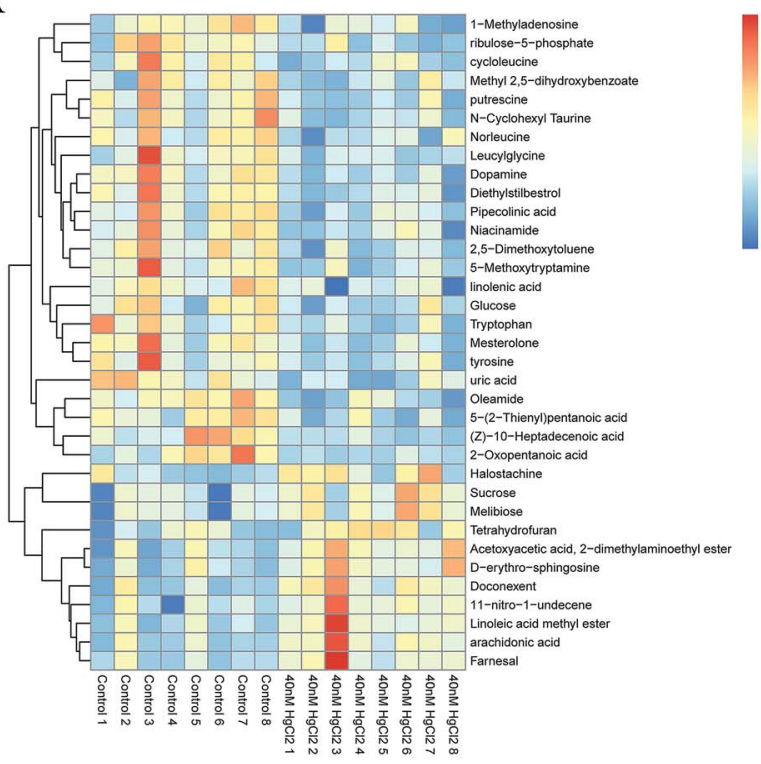

C

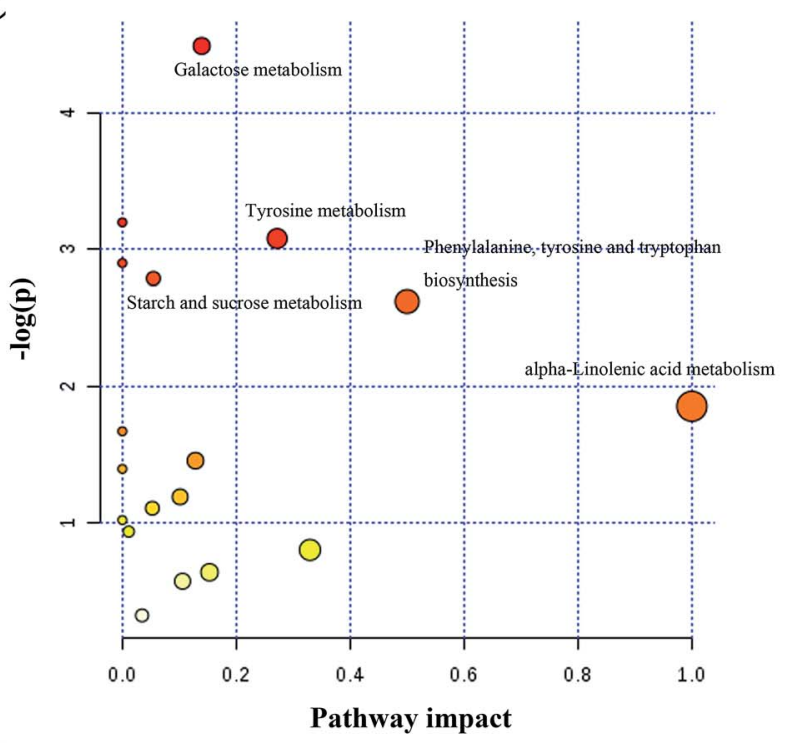

E

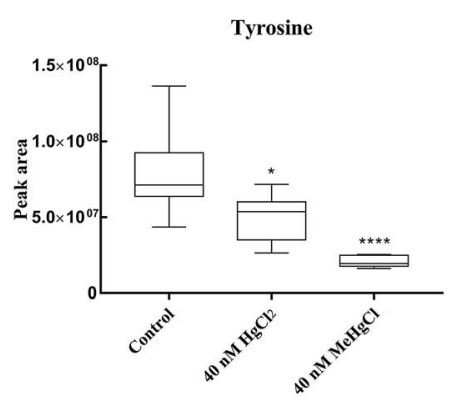

B

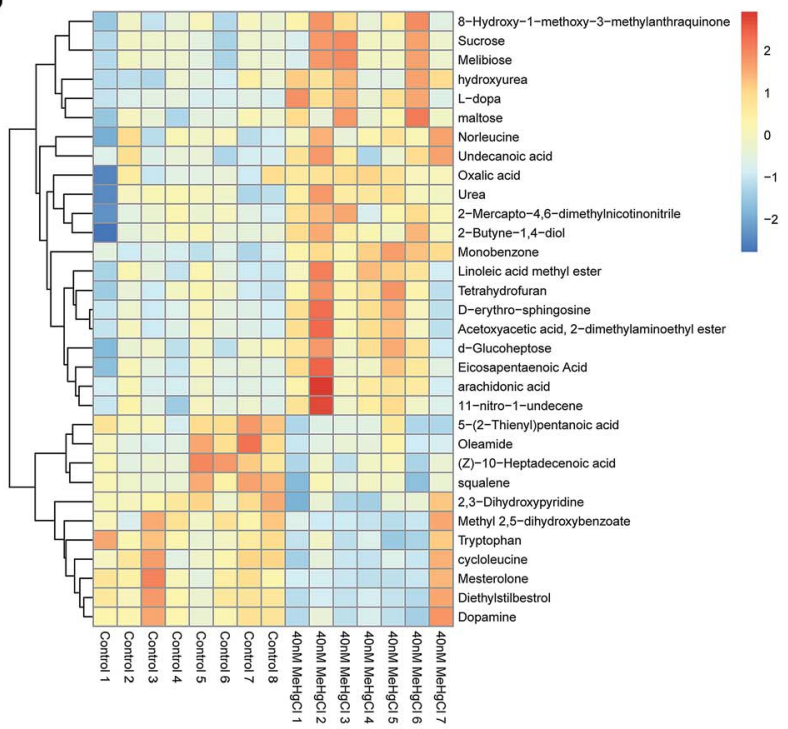

D

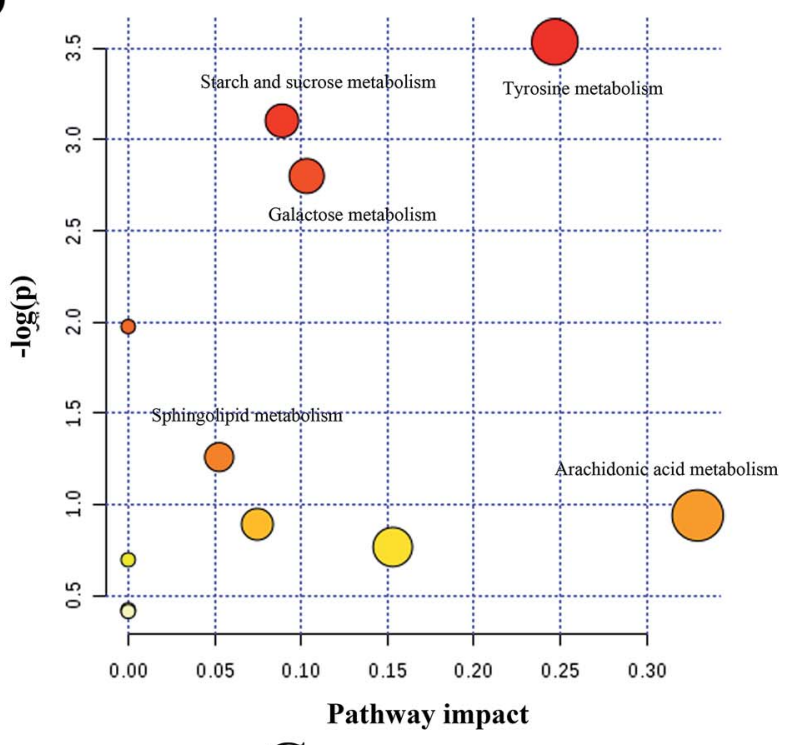

Dopamine

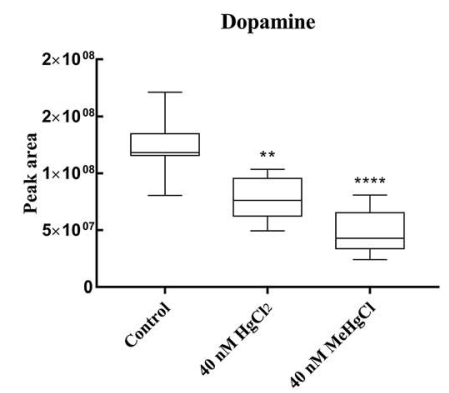

G

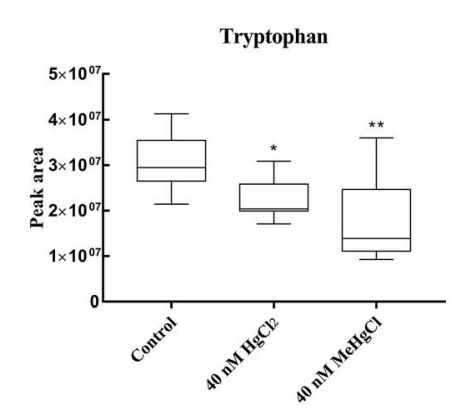

Fig. 4 Effects of mercury chloride $\left(\mathrm{HgCl}_{2}\right)$ and methylmercury chloride $(\mathrm{MeHgCl})$ on the metabolic profile. Heat map and hierarchical clustering analyses of zebrafish exposed to $40 \mathrm{nM}$ of $\mathrm{HgCl}_{2}(\mathrm{~A})$ and $\mathrm{MeHgCl}(\mathrm{B})$. Red colour represents an upregulation of the metabolite while blue color represents a downregulation. Relevant pathways disturbed by $\mathrm{HgCl}_{2}$ (C) and $\mathrm{MeHgCl}^{(D)}$. Bubble area is proportional to the impact of each pathway, with color denoting the significance from highest in red to lowest in white. Peak area of different metabolites across the different exposure groups at $30 \mathrm{hpf}$. The effect of $\mathrm{HgCl}_{2}$ and $\mathrm{MeHgCl}$ on (E) tyrosine, (F) dopamine, (G) tryptophan. Bars represent mean values ( $n=8$ ) and error bars represent standard deviations. $* P<0.05, * * P<0.01$; compared with control group. 


\section{Conclusions}

Our study demonstrated that $\mathrm{HgCl}_{2}$ exposure could delay the hatching period, impair the locomotor activity and increase apoptotic brain cell number in the zebrafish larvae, and these effects were aggravated in $\mathrm{MeHgCl}$ group. Their neurotoxicity may result from downregulation of neural development related genes, including $S h h, N g n 1$ and $N r d$. The metabolic profile showed that $\mathrm{HgCl}_{2}$ and $\mathrm{MeHgCl}$ could also disturb neurorelated tyrosine metabolism and phenylalanine, tyrosine and tryptophan biosynthesis. Furthermore, three neurotransmitters including tyrosine, dopamine and tryptophan were reduced after $\mathrm{HgCl}_{2}$ or $\mathrm{MeHgCl}$ exposure. Oxidative stress related to metabolite changes, such as putrescine, niacinamide, uric acid in $\mathrm{HgCl}_{2}$ group, whereas squalene in $\mathrm{MeHgCl}$. However, specific toxicological mechanisms of neurodevelopmental disorder induced by $\mathrm{HgCl}_{2}$ and $\mathrm{MeHgCl}$ need further explanation.

\section{Conflicts of interest}

The authors declare that there are no conflicts of interest.

\section{Acknowledgements}

This work was supported by the National Natural Science Foundation of China (81673228), and a project funded by the Priority Academic Program Development of Jiangsu Higher Education Institutions (2014).

\section{References}

1 Q. Shi, N. Sun, H. Kou, H. Wang and H. Zhao, Ecotoxicol. Environ. Saf., 2018, 164, 500-509.

2 J. S. Reif, A. M. Schaefer and G. D. Bossart, Vet. Sci., 2015, 2, 407-422.

3 L. The, Lancet, 2017, 390, 822.

4 S. Liang, Y. Wang, S. Cinnirella and N. Pirrone, Environ. Sci. Technol., 2015, 49, 3566-3574.

5 A. Dong, S. Zhai, P. Louchouarn, G. Izon, H. Zhang and X. Jiang, Environ. Sci. Pollut. Res. Int., 2019, 26, 4667-4679.

6 M. Liu, P. Du, C. Yu, Y. He, H. Zhang, X. Sun, H. Lin, Y. Luo, H. Xie, J. Guo, Y. Tong, Q. Zhang, L. Chen, W. Zhang, X. Li and X. Wang, Environ. Sci. Technol., 2018, 52, 124-134.

7 Q. Xu, L. Zhao, Y. Wang, Q. Xie, D. Yin, X. Feng and D. Wang, Environ. Pollut., 2018, 243, 115-126.

8 P. Li, Y. Pan, Y. Fang, M. Du, F. Pei, F. Shen, B. Xu and Q. Hu, Food Chem., 2019, 278, 587-592.

9 M. Korbas, T. C. Macdonald, I. J. Pickering, G. N. George and P. H. Krone, ACS Chem. Biol., 2012, 7, 411-420.

10 C. C. Bridges and R. K. Zalups, Arch. Toxicol., 2017, 91, 63-81. 11 T. Ke, F. M. Goncalves, C. L. Goncalves, A. A. Dos Santos, J. B. T. Rocha, M. Farina, A. Skalny, A. Tsatsakis, A. B. Bowman and M. Aschner, Biochim. Biophys. Acta, Mol. Basis Dis., 2018, DOI: 10.1016/j.bbadis.2018.10.024.

12 F. B. Teixeira, A. C. A. de Oliveira, L. K. R. Leao, N. C. F. Fagundes, R. M. Fernandes, L. M. P. Fernandes, M. C. F. da Silva, L. L. Amado, F. E. S. Sagica, E. H. C. de
Oliveira, M. E. Crespo-Lopez, C. S. F. Maia and R. R. Lima, Front. Mol. Neurosci., 2018, 11, 125.

13 G. Nedic Erjavec, M. Konjevod, M. Nikolac Perkovic, D. Svob Strac, L. Tudor, C. Barbas, T. Grune, N. Zarkovic and N. Pivac, Redox Biol., 2018, 14, 178-186.

14 A. K. Shukla, C. Ratnasekhar, P. Pragya, H. S. Chaouhan, D. K. Patel, D. K. Chowdhuri and M. K. R. Mudiam, Mol. Neurobiol., 2016, 53, 254-269.

15 F. Brandao, T. Cappello, J. Raimundo, M. A. Santos, M. Maisano, A. Mauceri, M. Pacheco and P. Pereira, Metallomics, 2015, 7, 1352-1363.

16 T. Cappello, F. Brandao, S. Guilherme, M. A. Santos, M. Maisano, A. Mauceri, J. Canario, M. Pacheco and P. Pereira, Sci. Total Environ., 2016, 548-549, 13-24.

17 K. N. Bridges, Y. Zhang, T. E. Curran, J. T. Magnuson, B. J. Venables, K. E. Durrer, M. S. Allen and A. P. Roberts, Environ. Sci. Technol., 2018, 52, 8774-8784.

18 M. M. Santoro, Trends Endocrinol. Metab., 2014, 25, 546-554. 19 M. Kamel and N. Ninov, Curr. Opin. Pharmacol., 2017, 37, 4150.

20 M. Zoupa and K. Machera, Int. J. Mol. Sci., 2017, 18, E817.

21 C. Zeng, H. Sun, P. Xie, J. Wang, G. Zhang, N. Chen, W. Yan and G. Li, Aquat. Toxicol., 2014, 149, 25-32.

22 P. Begley, S. Francis-McIntyre, W. B. Dunn, D. I. Broadhurst, A. Halsall, A. Tseng, J. Knowles, H. Consortium, R. Goodacre and D. B. Kell, Anal. Chem., 2009, 81, 7038-7046.

23 H. Zhao, H. Yan, L. Zhang, G. Sun, P. Li and X. Feng, Environ. Int., 2019, 126, 406-412.

24 P. H. Y. Chan, M. H. M. Chan, A. M. Li, R. C. K. Cheung, X. T. Yu and H. S. Lam, Environ. Res., 2018, 166, 418-426.

25 P. Grandjean and P. J. Landrigan, Lancet Neurol., 2014, 13, 330-338.

26 C. F. Huang, S. H. Liu, C. J. Hsu and S. Y. Lin-Shiau, Toxicol. Lett., 2011, 201, 196-204.

27 X. Xu, D. Weber, A. Martin and D. Lone, Neurotoxicol. Teratol., 2016, 53, 19-23.

28 I. W. Selderslaghs, J. Hooyberghs, R. Blust and H. E. Witters, Neurotoxicol. Teratol., 2013, 37, 44-56.

29 M. R. Karagas, A. L. Choi, E. Oken, M. Horvat, R. Schoeny, E. Kamai, W. Cowell, P. Grandjean and S. Korrick, Environ. Health Perspect., 2012, 120, 799-806.

30 S. Gentes, R. Maury-Brachet, C. Feng, Z. Pedrero, E. Tessier, A. Legeay, N. Mesmer-Dudons, M. Baudrimont, L. Maurice, D. Amouroux and P. Gonzalez, Environ. Sci. Technol., 2015, 49, 14560-14569.

31 C. Dou and J. Zhang, J. Hazard. Mater., 2011, 194, 277-282. 32 H. Li, S. Yu, F. Cao, C. Wang, M. Zheng, X. Li and L. Qiu, Ecotoxicol. Environ. Saf., 2018, 151, 1-9.

33 J. Kim, H. Oh, B. Ryu, U. Kim, J. M. Lee, C. R. Jung, C. Y. Kim and J. H. Park, Environ. Pollut., 2018, 236, 304-312.

34 T. Fukuda, A. Tokunaga, R. Sakamoto and N. Yoshida, Mol. Cell. Neurosci., 2011, 46, 614-624.

35 T. C. Tsai, C. C. Shih, H. P. Chien, A. H. Yang, J. K. Lu and J. H. Lu, BMC Dev. Biol., 2018, 18, 5.

36 H. Tu, T. Peng, J. Liu, X. Chen, C. Fan, Z. Huang, Y. Zhang, F. Zou and X. Meng, Aquat. Toxicol., 2018, 194, 167-175. 
37 B. Zhu, Q. Wang, X. Shi, Y. Guo, T. Xu and B. Zhou, Chemosphere, 2016, 144, 1646-1654.

38 A. Kim, C. Savary, C. Dubourg, W. Carre, C. Mouden, H. Hamdi-Roze, H. Guyodo, J. L. Douce and G. C. Frex Consortium, Brain, 2019, 142, 35-49.

39 T. S. Corman, S. E. Bergendahl and D. J. Epstein, Development, 2018, 145, dev167379.

40 M. B. Johnson, P. P. Wang, K. D. Atabay, E. A. Murphy, R. N. Doan, J. L. Hecht and C. A. Walsh, Nat. Neurosci., 2015, 18, 637-646.

41 J. Kim, C. Y. Kim, J. Song, H. Oh, C. H. Kim and J. H. Park, Neurotoxicol. Teratol., 2016, 54, 29-35.

42 A. N. Begum, J. S. Aguilar and Y. Hong, Environ. Res., 2017, 158, 194-202.

43 M. D. McCoole, N. J. Atkinson, D. I. Graham, E. B. Grasser, A. L. Joselow, N. M. McCall, A. M. Welker, E. J. Wilsterman
Jr, K. N. Baer, A. R. Tilden and A. E. Christie, Comp. Biochem. Physiol., Part D: Genomics Proteomics, 2012, 7, 3558.

44 M. Naderi, A. Jamwal, D. P. Chivers and S. Niyogi, Behav. Brain Res., 2016, 303, 109-119.

45 C. Gomez-Canela, D. Tornero-Canadas, E. Prats, B. Pina, R. Tauler and D. Raldua, Anal. Bioanal. Chem., 2018, 410, 1735-1748.

46 J. E. Montgomery, S. Wahlstrom-Helgren, T. D. Wiggin, B. M. Corwin, C. Lillesaar and M. A. Masino, Dev. Neurobiol., 2018, DOI: 10.1002/dneu.22606.

47 R. Godoy, S. Noble, K. Yoon, H. Anisman and M. Ekker, J. Neurochem., 2015, 135, 249-260.

48 N. Wang, J. Wei, Y. Liu, D. Pei, Q. Hu, Y. Wang and D. Di, Biomarkers, 2016, 21, 449-457. 